\title{
Mélanomes de la cavité buccale : présentation de 3 cas
}

\author{
Sofia Haïtami ${ }^{1}{ }^{*}$, Ishsane Ben Yahya ${ }^{1}$, Loubna Kinani ${ }^{1}$, Latefa Badr ${ }^{2}$ \\ ${ }^{1}$ Service d'0dontologie chirurgicale, Centre de Consultation et de Traitement dentaire, Casablanca, Maroc \\ ${ }^{2}$ Anatomopathologiste en pratique privée, Casablanca, Maroc
}

(Reçu le 5 juillet 2011, accepté le 8 septembre 2011)

\author{
Mots clés : \\ mélanome / \\ cavité buccale / \\ diagnostic / \\ pronostic / \\ traitement
}

Key words: melanoma / oral cavity / diagnosis / prognosis / treatment

\begin{abstract}
Résumé - Le mélanome est une tumeur qui se développe à partir des cellules pigmentaires dérivant des crêtes neurales. Les mélanomes buccaux constituent 0,4 à $8 \%$ de l'ensemble des tumeurs mélaniques. Ils atteignent plus l'homme entre 50 et 60 ans, et se développent essentiellement sur la muqueuse palatine, la gencive, les lèvres. L'étiopathogénie demeure inconnue; il existerait cependant une prédisposition génétique mais aucun précurseur bien défini n'a été isolé. Les microtraumatismes répétés sur un naevus bénin pré-existant ont également été incriminés. L'évolution du mélanome se fait en 2 phases : d'abord horizontalement, puis verticalement. La très haute malignité, l'agressivité et le sombre pronostic de cette tumeur en font une entité dont le diagnostic précoce permet d'améliorer de façon importante le taux de survie des patients. Nous présentons 3 cas de mélanomes dont le diagnostic tardif a rendu la prise en charge difficile et le pronostic sombre.
\end{abstract}

\begin{abstract}
Oral melanoma: 3 cases report. The melanoma is a tumor which develops from the pigmentary cells diverting neural crests. The oral melanomas constitute 0.4 to $8 \%$ of all the melanic tumors. They affect more the men between 50 and 60 years, essentially at the palatine mucous membrane, the gum, the lips. The etiopathogeny is unknown; there would be however a genetic predisposition but no well defined precursor was isolated. The repeated microtraumas on a pre-existing mild naevus were also incriminated. The evolution is made in 2 phases: at first horizontally then vertically. The very high wickedness, the aggressiveness and the somber forecast of these tumors make it entities in which the early diagnosis allows to improve the rate of survival of the patients. We present through this work 3 cases of oral melanomas; the late diagnosis maked the care difficult and the prognosis dark.
\end{abstract}

Le mélanome malin résulte de la transformation maligne des mélanocytes localisés dans l'épiderme, le derme ou la muqueuse et le chorion, plus rarement dans des viscéres. Les mélanomes de la cavité buccale constituent 0,4 à $8 \%$ de l'ensemble des tumeurs mélaniques [1]. Le diagnostic de mélanome est évoqué devant toute tumeur pigmentée de la peau ou des muqueuses qui présente une ulcération ou une surélévation.

L'objectif de ce travail est de présenter 3 cas de mélanomes de la cavité buccale dont l'évolution témoigne du caractère agressif et du mauvais pronostic de cette tumeur.

\section{Cas $n^{\circ} 1$}

Il s'agit d'une femme de 70 ans, consultant pour une tumeur maxillaire gauche indolore, apparue après une extraction dentaire réalisée 1 an auparavant. La patiente rapporte aussi une sensation d'étouffement.
L'examen clinique exo-buccal montre une asymétrie faciale avec une occlusion palpébrale gauche partielle (Fig. 1). La palpation révèle la présence d'adénopathies submandibulaires gauches. L'examen clinique endobuccal montre une tumeur ulcéro-bourgeonnante polylobulée, noirâtre, de consistance molle à la palpation, siégeant sur l'hémimaxillaire gauche, s'étendant dans le vestibule et sur le voile du palais (Fig. 2). La radiographie panoramique montre une lyse osseuse importante, intéressant toute la crête maxillaire gauche. L'examen tomodensitométrique met en évidence un processus ostéolytique agressif de l'hémiface gauche concernant la région fronto-éthmoïdo-maxillaire, avec envahissement des espaces profonds de la face (fosse nasale gauche, espaces pré et rétro-styliens, espace graisseux péri-orbitaire gauche...) (Fig. 3).

L'examen anatomopathologique confirme le diagnostic de mélanome. Le bilan d'extension met en évidence l'existence de

\footnotetext{
*Correspondance : haitamisofia@yahoo.com
} 


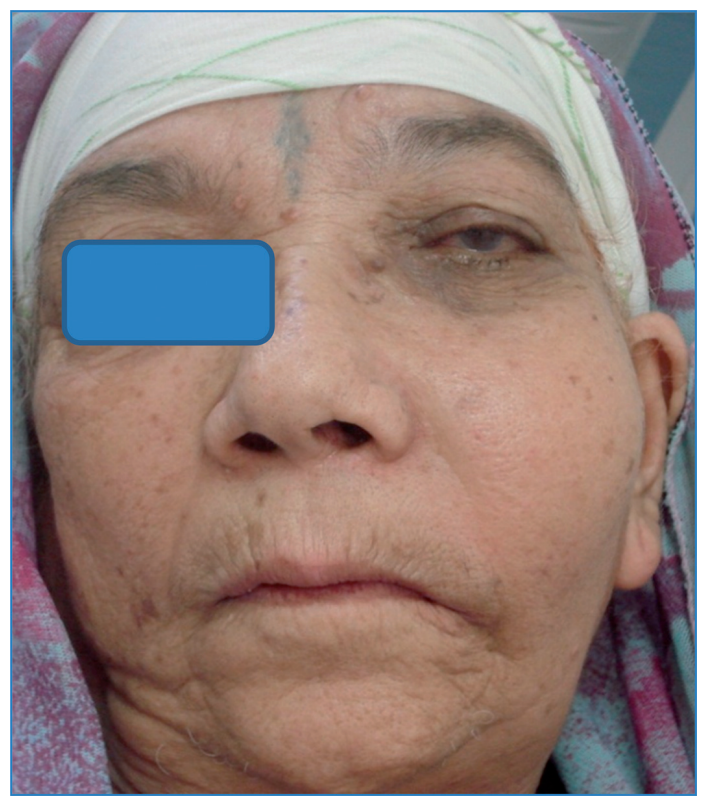

Fig. 1. Vue exobuccale: asymétrie faciale et occlusion palpébrale gauche partielle.

Fig. 1. Outer view: facial asymetry and partial left palpébral occlusion.

métastases. Le volume de la tumeur, l'existence de nombreuses métastases et l'âge de la patiente font opter pour l'abstention thérapeutique. La patiente décéde 9 mois plus tard.

\section{Cas $n^{\circ} 2$}

Il s'agit d'un homme de 84 ans qui consulte également pour une asymétrie faciale apparue après une extraction dentaire. L'examen exo-buccal montre un comblement du sillon nasogénien gauche avec, à la palpation, des adénopathies submandibulaires. L'examen endobuccal révèle une tumeur bourgeonnante, de couleur noirâtre, saignant au contact, siégeant sur la crête alvéolaire, allant de la 23 à la tubérosité maxillaire gauche avec extension des côtés vestibulaire et palatin. A sa surface, on note l'empreinte des dents (Fig. 4). La radiographie panoramique met en évidence une ostéolyse importante touchant le maxillaire gauche. L'examen tomodensitométrique montre que la tumeur envahit tout le palais gauche, sans atteindre l'orbite (Fig. 5).

L'examen anatomopathologique montre une prolifération mélanique maligne envahissant la muqueuse avec extension dans le chorion superficiel, confirmant le diagnostic de mélanome (Fig. 6).

Le patient est traité par exérèse chirurgicale (maxillectomie) et radiothérapie. Une prothèse obturatrice est réalisée. Le patient développe rapidement une récidive, puis il est perdu de vue.

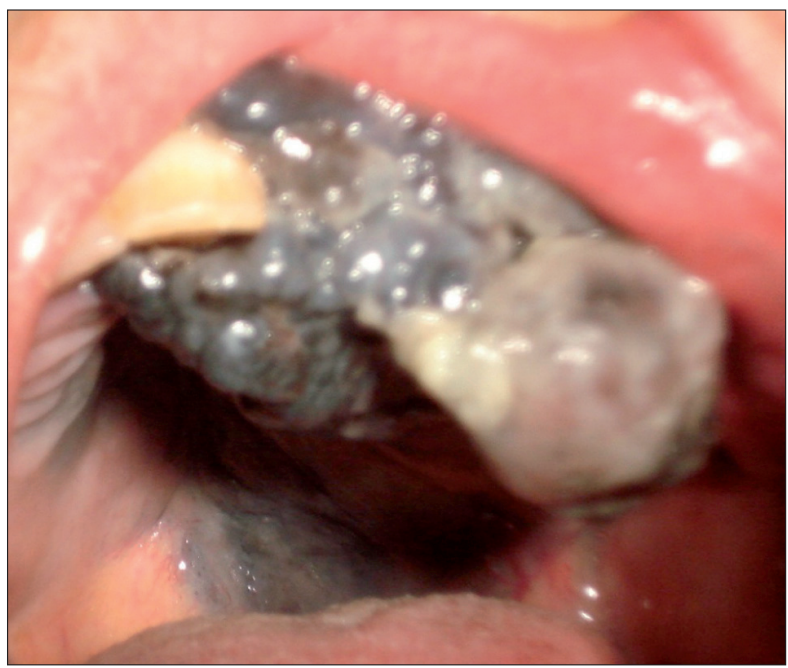

Fig. 2. Vue endobuccale : tumeur ulcéro-bourgeonnante polylobulée, molle, siégeant sur l'hémimaxillaire gauche et s'étendant dans le vestibule et sur le palais mou.

Fig. 2. Intra-oral view: ulcerative, buding, soft and multinodular tumor, localized on the left hemimaxilla, extending to the vestibule and the soft palate.

\section{Cas $\mathrm{n}^{\circ} 3$}

Un patient âgé de 60 ans se présente pour une tuméfaction mandibulaire vestibulaire d'aspect noirâtre qui serait apparue seulement depuis quelques jours.

L'examen exobuccal montre la présence d'adénopathies submandibulaires bilatérales. A l'examen endo-buccal, on retrouve un placard noirâtre siégeant sur la gencive mandibulaire gauche, allant de la région de la 35 au trigone rétromolaire, débordant du côté vestibulaire et du côté lingual (Fig. 7). La palpation révèle une induration dans la base de la langue.

L'imagerie médicale ne montre aucune atteinte osseuse (Fig. 8), et le bilan d'extension ne retrouve aucune métastase.

Un traitement conservateur est proposé au patient : il n'y a pas de résection mandibulaire, ni de curage ganglionnaire. L'exérèse chirurgicale est complétée par une radiothérapie. Quelques semaines après la fin de la radiothérapie, le patient présente une pleurésie. L'examen du liquide de ponction met en évidence des cellules métastatiques révélant l'existence de métastases pulmonaires. Une chimiothérapie est prescrite et le patient est actuellement encore en traitement.

\section{Discussion}

Le mélanome résulte de la transformation maligne des mélanocytes. Ces cellules dérivées des crêtes neurales siégent 


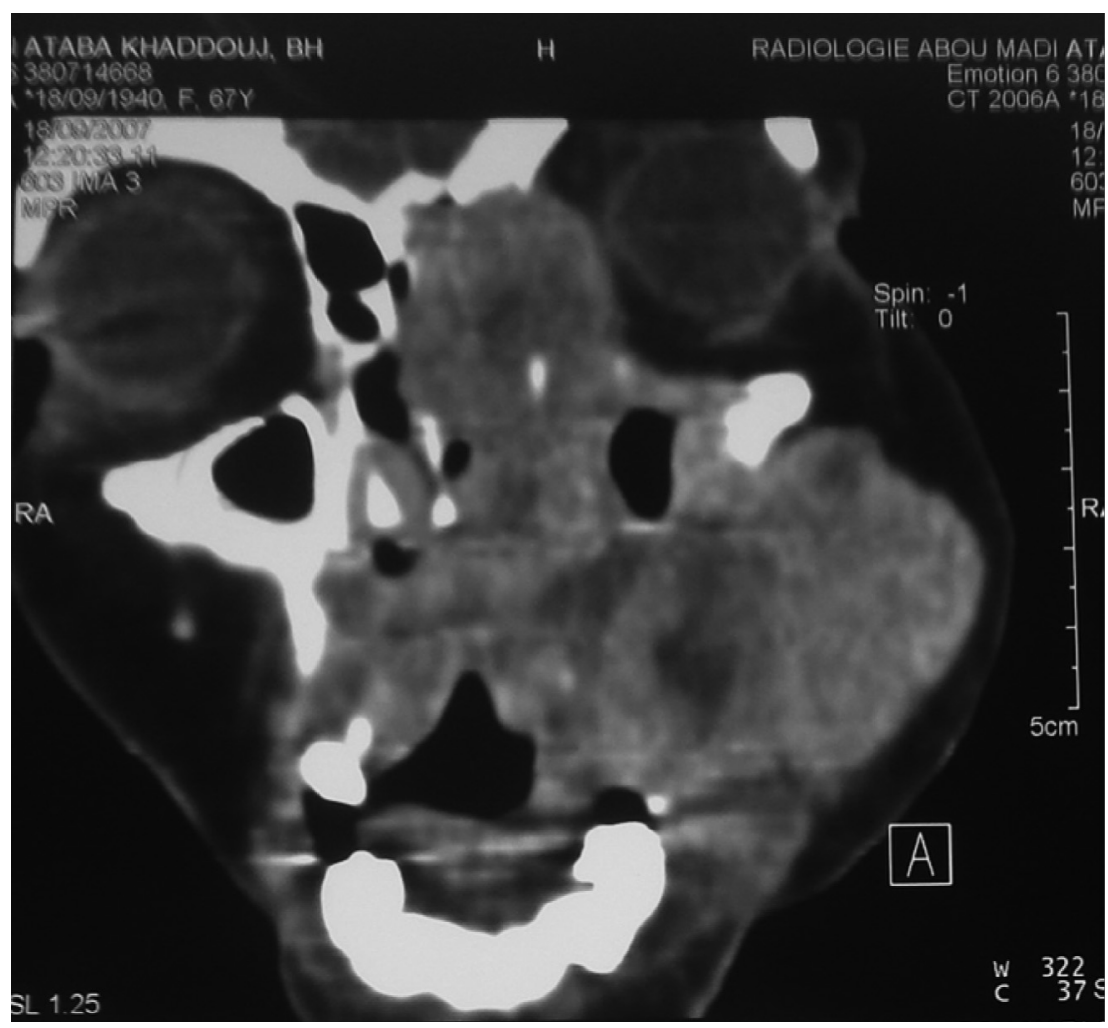

Fig. 3. CT-scan de la face en coupe coronale : processus ostéolytique agressif intéressant l'hémiface gauche (région fronto-éthmoïdo-maxillaire) avec envahissement des espaces profonds de la face.

Fig. 3. CT-scan of the face (coronal section): osteolytic and aggressive process interesting the left hemiface and invading the deep spaces of the face.

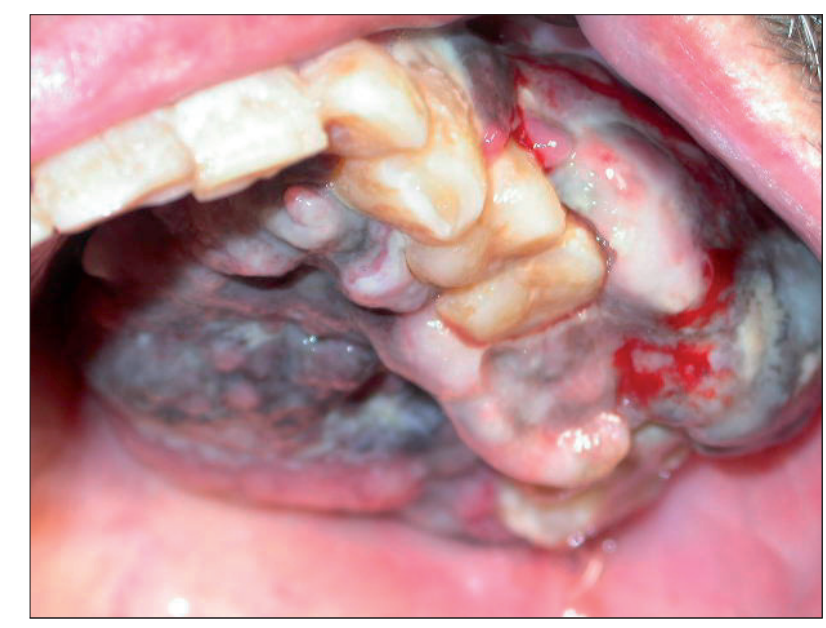

Fig. 4. Vue endobuccale: tumeur bourgeonnante, noirâtre, s'étendant de la 23 à la tubérosité maxillaire gauche, saignant au contact. Fig. 4. Intraoral view: black, buding tumor extending from 23 to the left maxillary tuberosity, bleeding on contact.

dans la couche basale de l'épiderme, le derme superficiel ou la muqueuse. Cette tumeur évolue en 2 phases: d'abord horizontalement, puis verticalement envahissant le chorion sous-jacent, les tissus mous et l'os, entrainant ainsi l'apparition de mobilités dentaires [3].

Le mélanome buccal est une tumeur rare qui représente 0,4 à $8 \%$ de l'ensemble des tumeurs mélaniques. Il est très invasif avec un degré d'agressivité et de malignité très élevé, d'où son mauvais pronostic. L'âge moyen de survenue se situe entre 50 et 60 ans, touchant beaucoup plus les hommes que les femmes ; 2 des 3 cas présentés ont été observés chez des hommes.

Dans la cavité buccale, le mélanome intéresse beaucoup plus le maxillaire que la mandibule [4]; dans les 3 cas présentés, 2 patients avaient une localisation maxillaire. Il siège le plus souvent sur la muqueuse palatine (40 à $50 \%$ des cas) avec une prédilection pour le palais dur (51\% des cas) ; le palais mou n'est touché que dans $8 \%$ des cas. La gencive supérieure est atteinte dans 20 à $30 \%$ des cas, la gencive inférieure dans 15 à $25 \%$ des cas. Il intéresse plus rarement les lèvres ( 8 à $10 \%$ des cas), les joues ( 4 à $6 \%$ des cas) ou la langue ( 2 à $3 \%$ des cas). Comme dans 2 des cas rapportés, la lésion peut évoluer dans un site extractionnel.

L'étiopathogénie est inconnue; il existerait cependant une prédisposition génétique mais aucun précurseur bien défini n'a été identifié. Les microtraumatismes répétés sur un naevus 


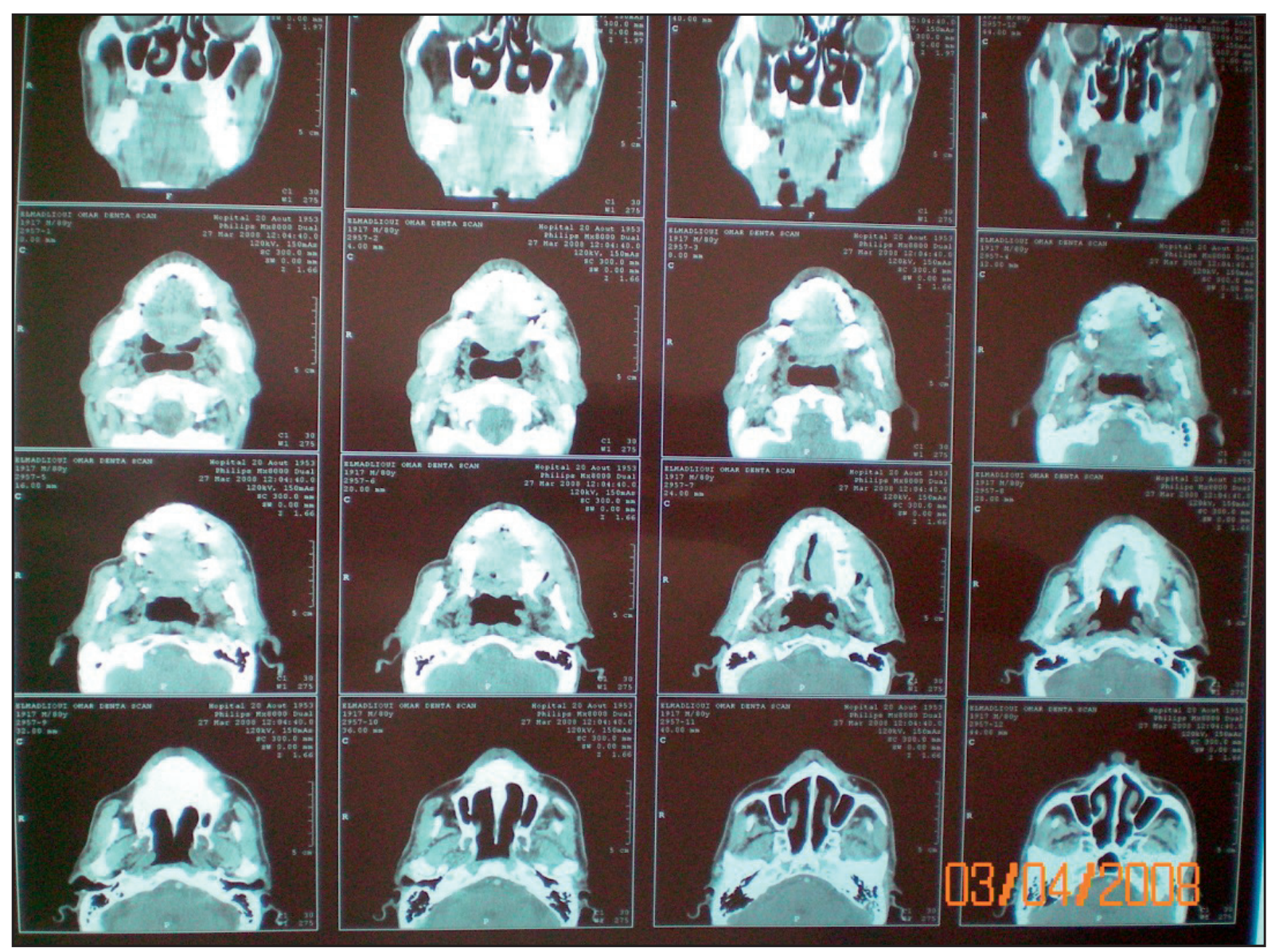

Fig. 5. CT-scan de la face : tumeur intéressant tout le palais gauche sans atteindre l'orbite.

Fig. 5. CT-scan of the face: tumor interesting the whole left palate without reaching orbit.

bénin pré-existant ont également été incriminés. Pour les mélanomes cutanés, le soleil et l'état de la peau jouent un rôle prédominant, d'où un risque augmenté pour les sujets à peau claire, s'exposant trop au soleil $[2,5]$.

Le diagnostic clinique est évoqué devant un nodule ou un placard plus ou moins étendu, de couleur non homogéne, allant du brun clair au noir anthracite, à surface irrégulière, souvent ulcérée et hémorragique.

L'examen anatomopathologique permet de confirmer le diagnostic devant l'existence de 3 critères considérés comme spécifiques: l'aspect des cellules tumorales (4 types possibles : pseudo-épithélial, fusiforme, indifférencié ou mixte), l'existence d'une activité jonctionnelle et la présence de mélanine dans les cellules tumorales. Dans les formes achromiques, le recours à l'immunohistochimie est indispensable.

Le diagnostic différentiel du mélanome se fait avec un tatouage à l'amalgame, une pigmentation physiologique ou tabagique, un granulome post-extractionnel, ou un mélanoacanthome... [6]. Certains auteurs proposent de frotter la lésion pigmentée avec une compresse : la teinte noirâtre se retrouve sur la compresse lorsqu'il s'agit d'un mélanome. La méthode serait fiable à 84,6 \% [7]. Le diagnostic différentiel avec un naevus bénin repose sur l'analyse morphologique selon les règles de L'ABCDairE modifié : le naevus devient Asymétrique avec des Bords irréguliers, de Couleur plus foncée ou inhomogène. L'évolutivité se traduit par le Diamètre de la lésion qui est supérieur à $6 \mathrm{~mm}$ ou qui augmente. L'Extension permanente de la lésion, qui change non seulement de taille, mais aussi de forme, de couleur et de relief, est également un critère pour le diagnostic différentiel entre un naevus et un mélanome [8].

Le pronostic des mélanomes est variable selon les différentes formes anatomocliniques et les localisations. Il est plus mauvais pour les mélanomes buccaux car, en l'absence des signes fonctionnels, la découverte est souvent tardive, c'està-dire en phase d'invasion verticale, souvent après qu'il ait déjà envahi le chorion profond et les vaisseaux. Le diagnostic du mélanome achromique est souvent très difficile, donc tardif, rendant le pronostic encore plus sombre [9].

Les mélanomes de la cavité buccale sont très lymphophiles : il existe des ganglions métastatiques dans $20 \%$ des 


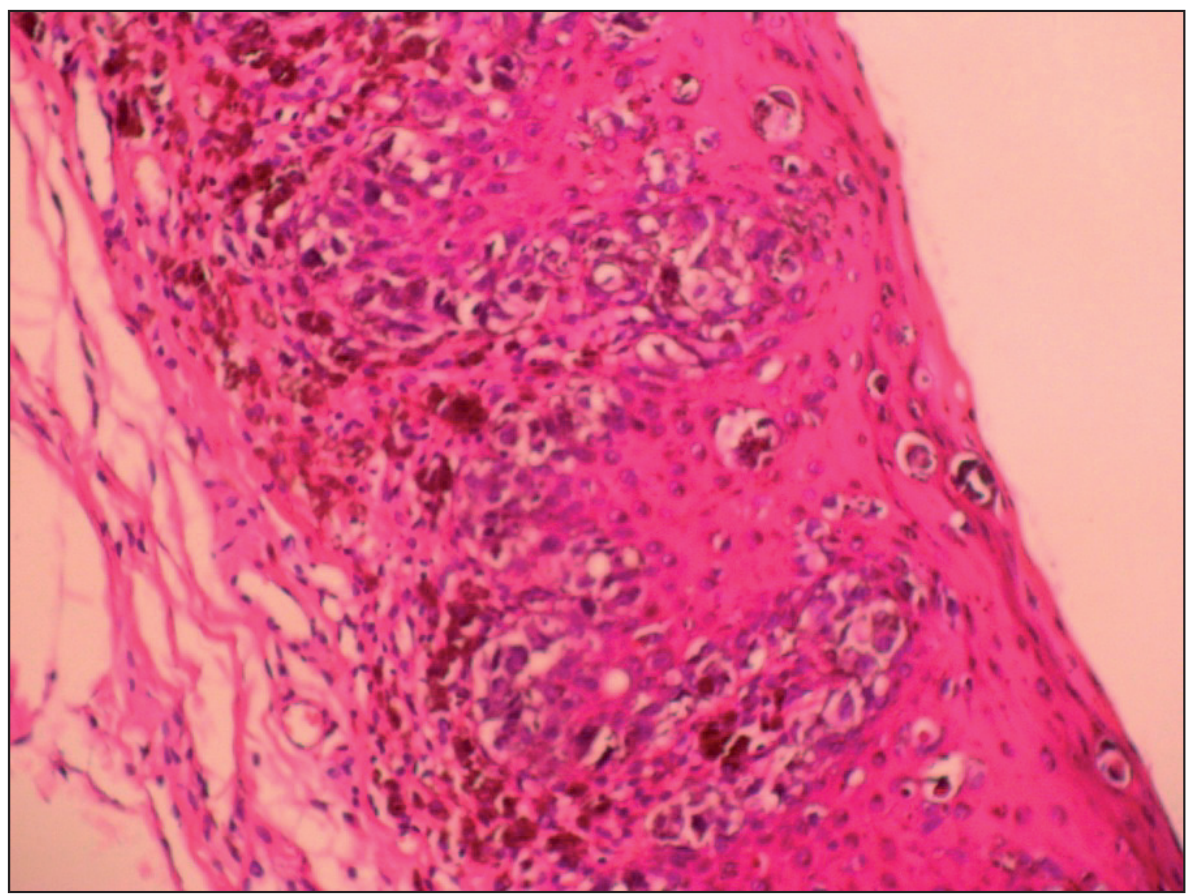

Fig. 6. Examen anatomopathologique : prolifération mélanocytaire maligne intéressant la muqueuse et envahissant le chorion superficiel. Fig. 6. Histological examination: melanotic malignant proliferation interesting the mucosa and invading the lamina propria .

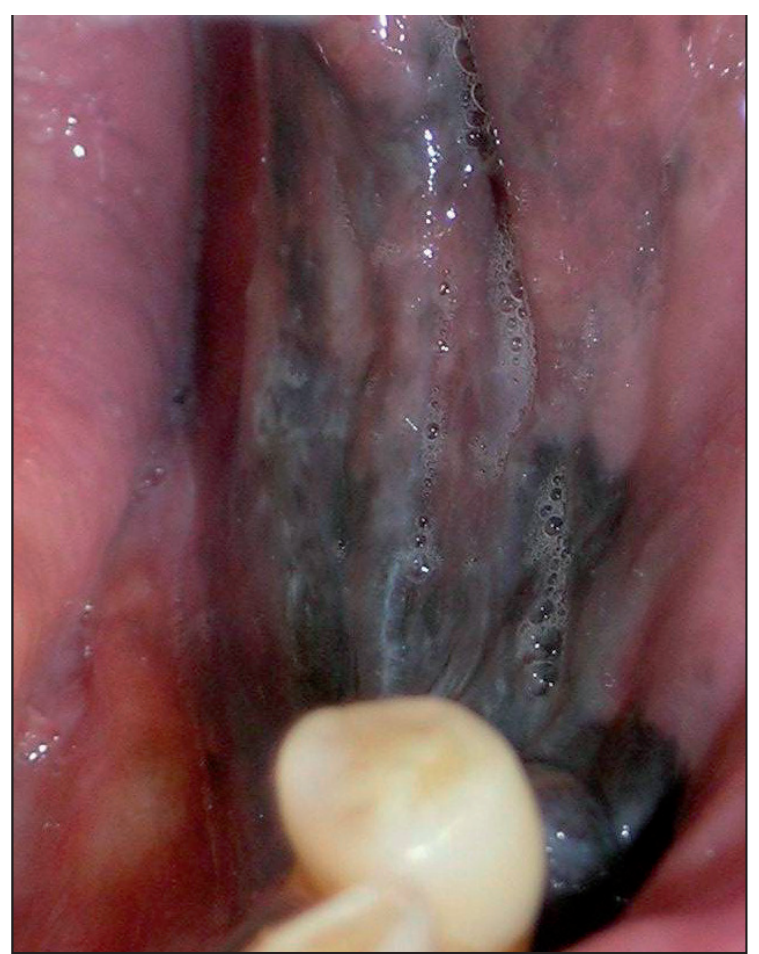

Fig. 7. Vue endobuccale : placard de couleur noirâtre, non homogène, intéressant la fibromuqueuse gingivale mandibulaire gauche, avec extension des côtés vestibulaire et lingual.

Fig. 7. Intraoral view: irregular blackish lesion on the left mandibular gingival with extension to the vestibular and lingual sides. cas lors du diagnostic. Ce pourcentage augmente avec l'épaisseur de la tumeur et assombrit le pronostic $[8,10]$.

$\mathrm{Au}$ stade tardif, devant une tumeur volumineuse et infiltrante, il est souvent impossible de préciser le point de départ et la variété du mélanome. S'il est détecté plus précocement, au stade intra-épithélial (mélanome in-situ) ou au stade initial de l'invasion du chorion, il est plus aisé de faire la distinction entre les diverses variétés, rendant le pronostic quelque peu différent bien que toujours sombre. Certains auteurs considèrent qu'il n'y a aucune corrélation entre les éléments précités et le pronostic de la tumeur [11, 12].

Exceptionnellement, il peut s'agir d'une métastase buccale d'un mélanome cutané (en particulier dans la région de l'amygdale et de la base de la langue) ou d'une extension à la cavité buccale d'un mélanome des fosses nasales ou des sinus maxillaires.

La survie moyenne des patients atteints d'un mélanome buccal est de 2 à 3 ans et le taux de survie est rarement de plus de $25 \%$ à 5 ans $[5,13]$.

Le traitement est basé sur l'exérèse chirurgicale associée ou non à une radiothérapie et à une chimiothérapie. Pour le $3^{\mathrm{e}}$ cas, il y a eu une chirurgie conservatrice associée à une radiothérapie, puis une chimiothérapie a été réalisée après la découverte des métastases pulmonaires [14, 15]. L'exérèse chirurgicale doit être la plus large possible, passant au moins à $1 \mathrm{~cm}$ des limites de la tumeur. Cette chirurgie radicale offre les meilleures chances de survie au patient $[16,17]$. Pour le $2^{\mathrm{e}}$ cas, une maxillectomie totale a été réalisée pour tenter 


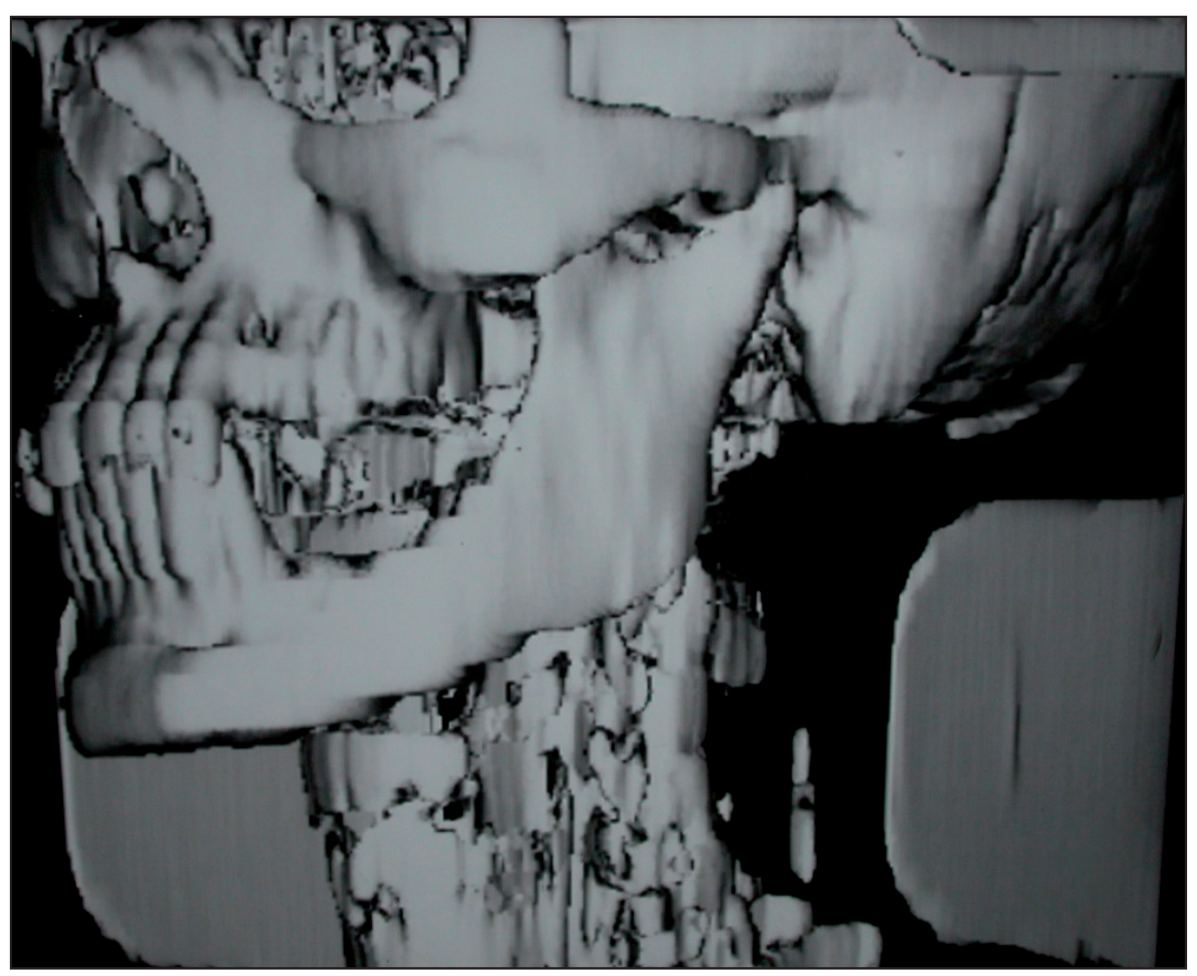

Fig. 8. Reconstruction tridimensionnelle : absence de lésions.

Fig.8. Three dimensionnal reconstruction: absence of lésions.

d'améliorer le pronostic. Certains auteurs préconisent un curage ganglionnaire systématique alors que pour d'autres, il est seulement indiqué devant la présence confirmée de ganglions métastatiques [18]. Certains se déterminent en fonction du ganglion sentinelle : ce premier relais ganglionnaire peut être détecté par injection de bleu de méthylène ou d'un radiotraceur autour du mélanome. Après son repérage, le ganglion est prélevé et examiné histologiquement : le curage est envisagé seulement si une métastase ou une micrométastase est décelée [19]. La radiothérapie peut être primaire pour les grosses tumeurs inopérables ou chez les patients âgés, ou complémentaire car elle permettrait un meilleur contrôle local sans modification significative de la survie $[20,21]$. Le $3^{\mathrm{e}}$ cas a bénéficié d'une radiothérapie complémentaire qui semble avoir été insuffisante pour éviter les métastases après une chirurgie conservatrice. La littérature rapporte également la possibilité d'associer chirurgie conservatrice et traitement au laser $\mathrm{CO}_{2}$ avec extraction des dents ayant un rapport avec la tumeur [22].

Le médecin dentiste occupe une place importante dans la prise en charge des mélanomes. Il joue un rôle non seulement dans le dépistage précoce des lésions pigmentées de la muqueuse buccale, mais aussi dans la prise en charge du patient au sein de l'équipe pluri-disciplinaire. Il intervient également dans la surveillance post-opératoire qui est primordiale et qui permet de diagnostiquer précocément l'apparition d'un deuxième mélanome, d'une récidive ou d'une métastase [23].
Conflits d'intérêt : aucun

\section{Références}

1. Aguas SC, Quarracino MC, Lence AN, Lanfranchi-Tizeira HE. Primary melanoma of the oral cavity : ten cases and review of 177 cases from literature. Med Oral Patol Oral Cir Bucal 2009;14: 65-71.

2. Hicks MJ, Flaitz CM. Oral mucosa melanoma: epidemiology and pathology. Oral Oncol 2000;36:152-69.

3. Patel S, Prasad ML, Escrig M, Singh B, Shaha AR, Kraus DH. Primary mucosal malignant melanoma of head and neck. Head Neck 2002;24:247-57.

4. Hoesrcsh B, Leiter U, Garbe C. Is head and neck melanoma a distinct entity? A clinical registry-based comparative study in 5702 patients with melanoma. Br J Dermatol 2006;155:771-7.

5. Patrick RJ, Fenske NA, Messina JL. Primary mucosal melanoma. J Am Acad Dermatol 2007;56:828-34.

6. Kauzman A, Pavone M, Blanas N, Bradley G. Pigmented lesions of the oral cavity: review, differential diagnosis and case presentations. J Can Dent Assoc 2004;70:682-3.

7. Wilson A. Delagado Azanero, Adalberto Mosqueda Taylor. Un método practico para el diagnostic clinic de melanomas de la mucosa oral. Med Oral 2003;8:348-52.

8. Duflo S, Monestier S, Zanaret M. Mélanomes malins cervicofaciaux. Encyc Med Chir, Paris : 20-950-E-10, 2008. 
9. Ulusal BG, Karatas 0, Yildiz AC, Oztan Y. Primary malignant melanoma of the maxillary gingiva. Dermatol Surg 2003;29:304-7.

10. Kienstra MA, Padhya TA. Head and neck melanoma. Cancer Control 2005;12:242-7.

11. Prasad ML, Patel S, Hoshaw-Woodard S, Escrig M, Shah JP, Huvos AG, Busam KJ. Prognostic factors for malignant melanoma of the squamous mucosa of the head and neck. Am J Surg Pathol 2002;26:883-92.

12. Mendenhall WM, Amdur RJ, Hineman RW, Werning JW, Villaret DB, Mendenhall NP. Head and neck mucosal melanoma. Am J Clin Oncol 2005;28:626-30.

13. Cebrian-Carretero JL, Chamorro-Pons M, Montes de Oca N. Melanoma of the oral cavity. Review of the literature. Med Oral 2001;6:371-5.

14. Medina JE, Ferlito A, Pellitteri PK, Shaha AR, Khafif A, Devaney $K 0$. Current management of mucosal melanoma of the head and neck. J Surg Oncol 2003;83:116-22.

15. Moreno MA, Hanna EY. Management of mucosal melanomas of the head and neck: did we make any progress? Curr Opin Otolaryngol Head Neck Surg 2010;18:101-6.

16. Loree TR, Mullins AP, Spellman J, North JH, Hicks WL. Head and neck mucosal melanoma: a 32-year review. Ear Nose Throat J 1999;78:372-5.
17. Penel N, Mallet $Y$, Mirabel X, Van JT, Lefevre JL. Primary mucosal melanoma of head and neck : pronostic value of clear margins. Laryngoscope 2006;116:993-5.

18. Gonzalez Garcia R, Naval Gias L, Martos PL, Nam-Cha SH. Melanoma of the oral mucosa. Clinical cases and review of the literature. Oral Surg 2005;0:264-71.

19. Garzino-Demo P, Fasolis M, Maggiore GM, Pagano M, Berrone S. Oral mucosal melanoma: a series of case reports. J Craniomaxillofac Surg 2004;32:251-7.

20. Temam S, Mamelle G, Marandas P, Wibault P, Avril MF, Janot F. Postoperative radiotherapy of primary mucosal melanoma of the head and neck. Cancer 2005;103:313-9.

21. Owens JM, Roberts DB, Myers JN. The role of postoperative adjuvant radiation therapy in the treatment of mucosal melanomas of the head and neck region. Arch Otolaryngol Head Neck Surg 2003;129:864-8.

22. Luna-Ortiz K, Campos-Ramos E, Pasche P, Mosqueda-Taylor A. Oral mucosal melanoma: conservative treatment including laser surgery. Med Oral Patol Oral Cir Bucal 2011;16:e381-5.

23. Gu GM, Epstein JB, Morton TH. Intraoral melanoma: long-term follow-up and implication for dental clinicians. A case report and literature review. Oral Surg Oral Med Oral Pathol 2003;96:404-13. 